\title{
De lo comunitario a lo escolar: elementos para el debate sobre la escuela comunitaria indígena
}

\section{Community and School. Elements for the Debate on the Indigenous Community School}

DOI: https://doi.org/10.32870/dse.v0i13.234

\section{Maike Kreisel*}

Resumen: Este trabajo aborda la experiencia de las Escuelas Secundarias Comunitarias Indígenas de Oaxaca, enfocándose en las relaciones que en la práctica pedagógica cotidiana se articulan entre conocimientos y prácticas comunitarias y escolares. Aunque apuntalado por las estructuras educativas oficiales, el modelo surge como alternativa pedagógica inserta en el movimiento oaxaqueño por la educación comunitaria y se fundamenta en la propuesta filosófica y etnopolítica de la comunalidad. A partir de un estudio etnográfico de larga duración realizado en una de las diez secundarias comunitarias, en el contexto de una comunidad zapoteca de la Sierra Norte de Oaxaca, este artículo reflexiona sobre algunas de las contribuciones, las problemáticas y los límites de proyectos que pretenden construir procesos educativos basados en el derecho de los pueblos originarios a la participación en la creación de sistemas educativos acordes a sus intereses culturales, lingüísticos y étnicos.

Las Secundarias Comunitarias aportan a ello desde procesos participativos, dialógicos y reivindicativos, en aras de una mayor justicia curricular, social y cognitiva, en un contexto marcado históricamente por desigualdades, discriminación y exclusión social. El modelo, en su intento de trascender las prácticas escolares asimilacionistas e integracionistas que han marcado la educación indígena en México, enfrenta tensiones que abarcan desde la construcción de procesos epistémicos plurales y la tendencia a la reificación y esencialización de lo cultural e identitario, hasta la reflexión acerca de la hibridación cultural, la apropiación de lo "ajeno" y la resignificación de lo "propio". En la práctica cotidiana cobran importancia las articulaciones y resignificaciones de las formas pedagógicas, escolares y comunitarias, además de la flexibilización de los espacios y tiempos del aprendizaje. Ante este panorama complejo, el modelo acierta en apostar por pedagogías más flexibles, dialogadas y co-ejercidas. Palabras clave: educación indígena, interculturalidad, participación de la comunidad, conocimientos, justicia.

\begin{abstract}
This article analyzes the experience of the Indigenous Community Secondary Schools of Oaxaca, Mexico, focusing on how alternative knowledge articulates with community and scholastic practices in everyday pedagogic activity. Although backed by official education agencies, the model emerged as a pedagogical alternative within Oaxaca's movement for a community education based on the philosophical and ethnopolitical proposal of the community. Based on a lengthy ethnographic study conducted in one of the 10 Indigenous Community Secondary Schools in a Zapoteco town in the northern sierra of Oaxaca, this article evaluates some of the contributions, problematics, and limits of the projects which aim to build educational processes based on the right of native peoples to participate in the creation of educational systems that respond to their cultural, linguistic, and ethnic interests.

The Community Indigenous Secondary Schools approach this goal through participation, dialogue, and cultural reclamation processes that seek to achieve greater curricular, social, and cognitive justice in a context of historical inequality, discrimination, and social exclusion. In its attempt to go beyond the integrationist and assimilationist educational practices that have characterized indigenous education in Mexico, the model faces numerous tensions, including those between the construction of alternative epistemes and the reification or essentialization of identity and culture, between the appropriation of what is "foreign" and the resignification of what is "ours," and those involved in the reflection on cultural hybridization. The articulations and resignifications of pedagogical, academic, and community forms become key in daily practice, as well as the flexibilization of learning spaces and times. Within this panorama, the model is successful in its search for more flexible, collaborative, and dialogic pedagogies. Key words: Indigenous education, interculturality, community participation, knowledges, justice.
\end{abstract}

* Doctorante en Investigación Educativa, Universidad Veracruzana.Correo electrónico: maike_k@yahoo.de 


\section{La escuela intercultural, territorio en disputa ${ }^{1,2}$}

Desde la creación de la Dirección General de Educación Indígena en el año 1978, la educación indígena se institucionalizó en México como parte de la oferta educativa oficial. Sin embargo, el tema de las características de una educación pertinente para los pueblos originarios, que desde tiempos del indigenismo ha sido objeto de debate, hasta la fecha sigue conformando un campo complejo, nutrido por diferentes enunciados yuxtapuestos que, aun enfatizando procesos de atención a la diversidad, articula sus discursos desde lugares pedagógicos, epistémicos y políticos distintos (Jiménez Naranjo, 2012).

Sería imposible entrar en este breve documento en los pormenores de esta discusión; ${ }^{3}$ no obstante, parece relevante resaltar que el modelo de las Secundarias Comunitarias Indígenas de Oaxaca que aquí exponemos es fruto de una serie de cuestionamientos a la histórica asimilación cultural que los pueblos originarios han sufrido a partir del proceso de escolarización. La introducción de la institución escolar en las comunidades, además, no ha contribuido a aminorar las relaciones asimétricas (de poder, económicas, epistémicas) de las que son sujetas en el presente (Walsh, 2005; Viaña, 2008; Santos, 2009).

Las Secundarias Comunitarias se plantean desde un posicionamiento crítico que pretende trascender las formas clásicas de la educación escolarizada. Podríamos vincular sus fundamentos discursivos a otro conjunto de propuestas - mexicanas, pero también muchas latinoamericanas- que buscan partir de la participación de los integrantes comunitarios en la definición de los contenidos y en las actividades del aprendizaje, retoman formas pedagógicas propias de las culturas originarias y flexibilizan tiempos y espacios de las actividades educativas de acuerdo a las condiciones comunitarias y retomando los ritmos del ciclo agrícola. El aspecto de la vinculación entre escuela y comunidad resulta central; se plantea "establecer un vínculo estrecho entre el conocimiento escolar y el familiar-comunitario en el medio indígena como eje fundamental" (Jiménez Naranjo, 2012: 176).

Encontramos propuestas como la red de escuelas wixárika y na'ayeri (De Aguinaga, 2010; Barba, Landey y Hernández, 2014) en Jalisco y Nayarit, las Telesecundarias Vinculadas a la Comunidad (Pieck, Messina y Colectivo docente, 2008) en el estado de Puebla, la experiencia de la UNEM en el estado de Chiapas (Sartorello, 2009; Bertely, 2011), las iniciativas de PRATEC en Perú por crear "una escuela amable con el saber local" (PRATEC, 2004), las experiencias de la Red de Educación Maya que ha promovido, entre otras iniciativas, una educación propia para los pueblos maya, xinka y garífuna en Guatemala (CNEM, 2013), o la red de escuelas impulsada por la fundación PROMIGAS en Colombia (Fundación PROMIGAS, 2010). Sus propuestas educativas son

1 Este trabajo es parte del proyecto de investigación "Las secundarias comunitarias indígenas de Oaxaca - resignificaciones de la educación escolarizada desde una propuesta alternativa para la formación de los jóvenes", en el doctorado en Investigación Educativa de la Universidad Veracruzana.

2 Agradezco a la doctora Yolanda Jiménez Naranjo, directora del trabajo doctoral en el que se basa el presente escrito, su revisión y aportaciones oportunas.

3 Sin embargo, hay muchos documentos que ofrecen panoramas globales o parciales sobre estos debates (Cfr. Hamel, 2001; Jiménez Naranjo, 2009, 2012; Sartorello, 2009;;Velasco y Jablonska, 2010; Bensasson, 2013; Dietz y Mateos, 2008, 2011; Martínez Buenabad, 2015). 
De lo comunitario a lo escolar: elementos para el debate sobre la escuela comunitaria indígena

pedagógicas y curriculares, pero también políticas en los términos antes descritos. Son iniciativas promovidas por miembros de la academia de asociaciones civiles y por organizaciones, intelectuales y ciudadanos de los pueblos originarios y afrodescendientes.

Las prácticas educativas en dichas propuestas, sin embargo, no se encuentran libres de tensiones, pues se sitúan en un contexto sociohistórico y político complejo en el que inciden tanto las trayectorias de los actores escolares, docentes y estudiantes como las biografías de las mismas comunidades de los pueblos originarios, inmersas en procesos de aculturación, discriminación, racismo y resistencia, en su relación con la sociedad mayoritaria (Walsh, 2005: 32). A ello ha contribuido históricamente la misma educación escolarizada, que ha pretendido convertir las culturas indígenas a otras formas de vida, catalogadas por algunos como de mayor futuro. Innegablemente también ha influido en ello el inevitable proceso de diálogo con espacios socioculturales más amplios y diversos, la apropiación de elementos culturales ajenos y una continua hibridización de las prácticas sociales, como transformaciones que ocurren habitualmente y que son muestra del carácter dinámico y rearticulador de los procesos culturales (García Canclini, 2001).

Comprendemos aquí la apropiación cultural como un proceso permanente "que obedece a factores internos y/o externos y que se traduce en la creación o apropiación de bienes culturales de diversa naturaleza (materiales, de organización, de conocimiento, simbólicos, emotivos)" (Bonfil Batalla, 2004: 119). A través de la apropiación, algunos elementos procedentes de otros contextos culturales son integrados a la cultura propia. En sus articulaciones concretas, juegan un papel importante los conflictos sociales y las relaciones de poder que caracterizan el contexto y las dinámicas interétnicas. Bonfil Batalla interpreta estas tensiones en términos de una "lucha por el control cultural" - conllevan, de esta manera, también procesos de innovación de bienes culturales propios y la resistencia a elementos ajenos- (Bonfil Batalla, 1991:185). Parece relevante reconocer que la apropiación cultural, aunque limitada por el "carácter coactivo... de la herencia cultural", conlleva "un sentido de la naturaleza activa y transformadora del sujeto... [que] sitúa claramente la acción en las personas que toman posesión de los recursos culturales disponibles y los utilizan" (Rockwell, 2005: 29).

Consideramos que estas tensiones se encuentran presentes también en los procesos de apropiación de la institución escolar por los proyectos educativos alternativos de los pueblos originarios; se relacionan tanto con cómo se interpreta el enfoque intercultural y comunitario en discurso y práctica, como también con los complejos procesos de reificación y esencialización cultural e identitaria que pudieran emerger en ellos, y que son imposibles de analizar de forma estática y unívoca (Jiménez Naranjo, 2012). Se configuran en el marco de la resistencia de los pueblos originarios frente a la sociedad mayoritaria. Dietz aduce, remitiéndose a Bonfil Batalla (1987), que "como resultado de la etnificación de las prácticas culturales, éstas se constituyen en la 'cultura propia' de un determinado grupo", donde la "identificación étnica" se utiliza como instrumento de reivindicación (Dietz, 1999: 64). Es así como la apropiación de la institución escolar desde planteamientos 
reivindicativos forma parte también de procesos de etnogénesis (Dietz, 1999: 65; Bertely, 2005; González, 2006).

Un reto que Jiménez Naranjo (2012) detecta para los proyectos educativos alternativos -en relación a estas tensiones- es incorporar procesos de reflexión a nivel metacultural, ${ }^{4}$ para analizar cómo y en qué medida es posible relacionar las prácticas culturales como tales con el currículum escolar. Revisaremos cómo en el caso de las Secundarias Comunitarias Indígenas de Oaxaca se perfila el diálogo con las prácticas culturales en la cotidianidad de una propuesta educativa que se comprende en estrecha vinculación con la comunidad y sus saberes y pretende ir más allá de la incorporación de elementos culturales folclorizados al aula.

Para ello, recuperamos algunos de los hallazgos de un estudio etnográfico (Rockwell, 2011; Velasco y Díaz de Rada, 1997) realizado en una comunidad zapoteca de la Sierra Norte de Oaxaca, en el que se buscó conocer cómo se articula la práctica educativa del modelo en un contexto concreto. A través de una estancia de larga duración, se participó en los quehaceres escolares cotidianos y la vida comunitaria; las opciones metodológicas fueron la observación participante, las entrevistas semi-estructuradas y abiertas, y los grupos de discusión. Además, se buscó el diálogo con diferentes actores, académicos e institucionales, relacionados con la propuesta y la educación indígena en el estado. Para el análisis de los datos se construyó un sistema de categorización (Díaz de Rada, 2011), pretendiendo generar un proceso dialéctico entre las categorías emergentes desde el material empírico, y los elementos teóricos que brindaron insumos para su interpretación, para lo cual se fueron alternando fases de trabajo de campo y de sistematización.

\section{Las Secundarias Comunitarias: una propuesta alternativa para la educación de los pueblos originarios}

El modelo de las Secundarias Comunitarias Indígenas de Oaxaca surge como parte de las iniciativas por una educación comunitaria pertinente y dialógica, promovidas por el Movimiento Pedagógico de la Coalición de Maestros y Promotores Indígenas de Oaxaca (CMPIO); nace como fruto de los esfuerzos del magisterio y en diálogo con comunidades, académicos, intelectuales indígenas y no indígenas, e instituciones interesadas. Se suscribe a la corriente de la educación comunitaria oaxaqueña y el pensamiento de la comunalidad, como filosofía y modo de vida de las comunidades originarias de Oaxaca (Rendón Monzón, 2003, 2011; Martínez Luna, 2013; Díaz, 2007; Maldonado, 2010).

El modelo, validado a través de un acuerdo entre la Sección XXII del SNTE-CNTE y el Instituto Estatal de Educación Pública de Oaxaca (IEEPO), comenzó a trabajar en el año 2004, con una fase de pilotaje con las primeras cinco escuelas, para su posterior evaluación. Actualmente cuenta con

4 Jiménez Naranjo entiende el concepto de "meta-cultura" como el "proceso que se genera cuando empleamos la cultura para reflexionar sobre ella de forma deliberada". Estos procesos poseen un mayor nivel de abstracción que los culturales, sin por ello ser identitarios (Jiménez Naranjo, 2012: 181). 
diez planteles insertos en diferentes regiones indígenas del estado. Apuesta por una metodología propia que prescinde de la fragmentación de los campos de conocimientos por disciplinas; en su lugar, promueve el desarrollo de proyectos de aprendizaje e investigación sobre temas relevantes para la comunidad, en los cuales fomenta el diálogo entre los conocimientos y prácticas comunitarias y los contenidos escolares. Cada generación de estudiantes está a cargo de un educador que orienta los procesos formativos que pretenden impulsar el aprendizaje autónomo y colaborativo. ${ }^{5}$ Busca fortalecer la participación de padres de familia e integrantes comunitarios en los procesos de aprendizaje y en la toma de decisiones escolares. A continuación, profundizaremos en el análisis de la práctica observada en una de las diez escuelas. Proyectamos esta reflexión a través de dos ejes: el diálogo de conocimientos ${ }^{6}$ y la interlocución entre prácticas pedagógicas comunitarias y escolares.

\section{Dialogando con lo ajeno y resignificando lo propio 7}

La Secundaria Comunitaria estructura su práctica educativa a partir de temas de interés local. El currículum se define procesualmente en cada generación con base en los proyectos de investigación y aprendizaje; estos proyectos buscan la recuperación de los conocimientos locales, para poder dialogar con los saberes disciplinarios (ESCI/IEEPO, 2007). Esta lógica implica un reposicionamiento epistemológico, deconstruyendo los esquemas tradicionales de la educación escolarizada que generalmente permanece inserta en la discursividad occidental moderna (López Pérez, 2004: 205), ${ }^{8}$ a pesar de que se fomente, desde la mirada constructivista, cada vez más la contextualización y significación situada del aprendizaje (Lave y Wenger, 1991; Díaz Barriga, 2003).

En México y en diferentes países de América Latina, en las últimas décadas, tanto desde el análisis académico como también en diferentes iniciativas innovadoras de educación comunitaria e

5 Los educadores de las Secundarias Comunitarias provienen de distintas comunidades originarias del estado. Al contar el modelo únicamente con el reconocimiento a nivel estatal, pero no federal, no dispone de un presupuesto propio, situación que frente a la reforma educativa se ha agravado. Los docentes no cuentan con las percepciones correspondientes al nivel educativo, laborando, no obstante, en horarios ampliados debido a la flexibilidad de tiempos que requiere el trabajo por proyectos. Muestran altos niveles de compromiso con su labor, aun al enfrentar una importante situación de incertidumbre bajo las actuales tensiones políticas que ponen en juego las condiciones de la educación comunitaria oaxaqueña. Esto, a pesar de encontrarse respaldada por el PTEO y la reciente Ley de Educación del estado de Oaxaca, decretada por el congreso estatal el pasado mes de abril. Esta última reconoce a las Secundarias Comunitarias como parte de la oferta educativa del estado (art.16).

6 Aunque conscientes que autores como Enrique Leff (2004) y Boaventura de Sousa Santos (2010) se refieren más bien a saberes al abordar la interlocución entre culturas, no distinguimos aquí, desde el punto de vista analítico, entre los conceptos de saber y conocimiento, ya que en la práctica de las Secundarias Comunitarias se emplean ambos términos. Así, uno de los asesores explica que el modelo curricular busca crear "vínculos entre el conocimiento comunitario y el conocimiento disciplinario para establecer una especie de diálogo de culturas" (Ob-transcripción-curso de formación docente).

7 Bonfil Batalla plantea que los elementos culturales son propios cuando han sido recibidos "como patrimonio cultural heredado de generaciones anteriores" que se siguen produciendo, manteniendo y transmitiendo por el grupo social en cuestión. Los elementos ajenos, en cambio, "forman parte de la cultura que vive el grupo, pero que éste no ha producido ni reproducido" (Bonfil Batalla, 1991: 7). Asimismo desarrolla, como hemos mencionado, el concepto de apropiación cultural designando la integración de elementos considerados como ajenos a la cultura propia. Es para fines de análisis que incurrimos, en cierta medida, en la dicotomización de la cultura propia y ajena, aunque estamos conscientes de la heterogeneidad e hibridez de los procesos culturales. Reconocemos que "el uso de esta dicotomía propio-ajeno o local-universal, como categorías de delimitación identitaria, ciertamente esconden una profunda heterogeneidad interna no explicitada y potencialmente conflictiva" (González Apodaca, 2006: 230).

8 López Pérez analiza el discurso de la educación en México, constatando que este "puede ser leído también desde el llamado pensamiento moderno occidental, entendido no sólo como la expansión de occidente desde el siglo XVI, religión y economía, sino también como forma hegemónica de conocimiento, considerada como la única manera de pensar un mundo" (López Pérez, 2004: 205). 
intercultural, se ha impulsado una visión crítica de los trasfondos epistemológicos de la educación, destacando, entre otros elementos, la búsqueda de una mayor justicia cognitiva (Santos, 2007: 53). Algunos autores latinoamericanos han abogado por reconocer la presencia de la "colonialidad del saber" (Castro-Gómez, 2000) en el ámbito escolar, en las universidades, en las lógicas de progreso y movilidad social, y de esta forma, en diferentes áreas de la vida cotidiana. Daniel Mato constata al respecto que la tendencia a dividir el conocimiento de manera dicotómica en uno "poseedor de verdades 'universales' y el otro sólo de verdades 'locales' es tan antigua como el credo en la superioridad de la "civilización occidental"” (Mato, 2008: 102).

A partir del análisis de experiencias de investigación en proyectos educativos emergentes, González Apodaca y Rojas señalan que la "incorporación de los saberes comunitarios o conocimientos indígenas a los procesos de enseñanza-aprendizaje de las escuelas, y su articulación con los conocimientos escolares convencionales" (González y Rojas, 2013: 292) se constituye como uno de los retos mayores para el futuro de la educación intercultural. El diálogo de saberes (Leff, 2004), en la práctica, implica replantear el quehacer educativo de la institución escolar y, por parte de los docentes, el desarrollo de nuevos saberes pedagógicos. Tovar aduce que no se trata de una simple traducción, pues unos y otros saberes se basan en racionalidades distintas, entendidas como "sistemas culturales de interpretación que reflejan las concepciones y saber de colectivos sociales" (Tovar, 2009: 4-5). El saber se conforma, a partir de prácticas colectivas y en un contexto concreto, como saber situado; es así como podemos hablar de una pluralidad de epistemologías (Tovar, 2009: $6)$.

Una pregunta central sería, por ende, cómo crear espacios adecuados para forjar nuevas prácticas pedagógicas mediante las que pueda entablarse el diálogo entre, "a la vez, los conocimientos indígenas en su forma viva y practicada en la comunidad y el conjunto de los contenidos escolares convencionales" (Gasché, 2004: 198), sin perder de vista los contextos de los que provienen, teniendo en cuenta la genealogía de cada discurso (Dietz y Mateos, 2011: 121). Rengifo argumenta que el diálogo de saberes ha de efectuarse explicitando "que el conocimiento de la escuela es otro y que pertenece a una visión del mundo diferente al de la cosmovisión andina, y que el trato entre ambas debe tener como referencia la equivalencia y no la jerarquía" (Rengifo, 2003: 113).

Una tendencia de la práctica educativa -e incluso del discurso académico, como analizan Dietz y Mateos (2011) - puede ser la dicotomización de los saberes, es decir, su concepción como dos entidades separadas y la tendencia a su reificación. Dietz y Mateos argumentan que para los pueblos originarios esta dicotomización puede resultar provechosa en términos políticos, además de ser propiciada por mismas distancias geográficas de los espacios urbanos en las que muchas comunidades originarias se localizan (Dietz y Mateos, 2011: 134). De esta manera, la articulación de los saberes representa un reto de interpretación y creación de estrategias para los actores educativos. 
En la investigación realizada en el modelo de las Secundarias Comunitarias Indígenas de Oaxaca, se observó la tendencia de los actores a definir el papel de la Secundaria Comunitaria en relación al rescate de la cultura propia que se percibe como amenazada, históricamente, por los procesos de aculturación.

Lo que se trata, de rescatar, trabajar muy fuertemente con lo comunitario porque en muchos lugares se están perdiendo muchas cosas. ... desde ahí hay que darle más prioridad a lo que es comunitario porque pues es propio, es algo de nosotros que nos han dejado que desde siempre se ha practicado con nuestros abuelos (E-Elena-educadora).

Frente a las experiencias de discriminación étnica, lo que se considera como parte de las influencias de la cultura mestiza o urbana en ocasiones se analiza en el contexto escolar más bien en relación a sus desventajas, en comparación con las prácticas culturales consideradas como propias y que se ven en riesgo de ser desplazadas. Interpretamos esta tendencia como parte de los procesos de resistencia ante las interrelaciones asimétricas entre culturas, que conllevan la necesidad de reivindicar "lo propio" frente a "lo ajeno". En el caso del siguiente ejemplo, los estudiantes contrastan a los espacios comunitarios y citadinos:

En la comunidad dicen que la forma de pensar de la comunidad es diferente a una ciudad, ¿por qué?, porque en la ciudad cuidan menos el medio ambiente y mientras que en la comunidad cuidan y aprovechan más lo natural como son las plantas y los animales, en la comunidad consumen lo que siembran, por ejemplo el maíz y el frijol son unas de las plantas más sembradas en la comunidad, en cambio los que viven en la ciudad nada más piensan en conseguir dinero, a costa del daño que hacen a la naturaleza. En la comunidad, conforme pasa el tiempo ha habido cambios en la forma de pensar, por ejemplo, si comparamos la forma de pensar de un anciano y la de un joven los resultados no serían iguales, estos cambios en la forma de pensar son resultado de la contaminación ideológica que se está dando en nuestra comunidad (Doc-trabajo de investigación-4ta generación).

La secundaria comunitaria busca fortalecer la cultura e identidad originarias; ${ }^{9}$ la contrastación establecida entre determinadas prácticas culturales, propias y ajenas, tiende a etnificar a las prime-

9 Para nuestra comprensión del concepto de cultura nos basamos en autores como Rogoff, Moore, Correa-Chávez y Dexter, que entienden la cultura en términos de "repertorios de práctica”; esta concepción nos lleva a transitar hacia una noción de la cultura como experiencias vividas por las personas a lo largo de su vida, a través de las mismas prácticas sociales e inmersas en la misma historia comunitaria (Rogoff et al., 2015: 491). Giménez señala que, sin embargo, "la cultura nunca debe entenderse como un repertorio homogéneo, estático e inmodificable de significados" (Giménez, 2009: 10). La cultura, de esta manera, se transforma, sujeta a hibridaciones a través del contacto cultural. Por otra parte, comprendemos las identidades como "repertorios culturales interiorizados .... a través de los cuales los actores sociales (individuales o colectivos) demarcan sus fronteras y se distinguen de los demás actores en una situación determinada, todo ello dentro de un espacio históricamente específico y socialmente estructurado" (Giménez, 2000: 54). Entendemos que las identidades no se constituyen a partir de características fijas, sino que son procesuales y plurales; Dietz plantea, en este sentido, una visión de "carácter diverso, plural, multi-situado, contextual y por ello necesariamente híbrido de las identidades culturales, étnicas, de clase, de género" (Dietz, 2013: 184). 
ras como parte de la reivindicación. Richard plantea en este sentido el concepto de "identidad por oposición", proceso en el que se dicotomiza al "nosotros" frente a "los otros" en relación a determinadas características (Richard, 1993: 212). Escárcega hace énfasis en el peso político que tiene la esencialización estratégica de las identidades como parte de la lucha de los pueblos originarios por una mayor justicia social (Escárcega, 2009: 43). Un riesgo de la esencialización sería obviar la heterogeneidad al interior del grupo cultural (Dietz, 2013: 179); en nuestro caso, advertimos cómo, sin embargo, en el análisis de la cultura comunitaria está presente la mirada de lo procesual y de los cambios generacionales, más que una concepción estática de la cultura. Estos cambios son percibidos, en este caso, como una "contaminación" de lo propio a través del sesgo que les imprime la perspectiva reivindicativa fomentada en la práctica.

En cuanto a la aproximación al diálogo entre conocimientos, Escárcega propone partir del reconocimiento de la cultura propia para pensar en la apropiación de otros elementos culturales (Escárcega: 2009: 43). Esta apropiación pasa por la mirada crítica del análisis de la genealogía del discurso del "otro" conocimiento, y en este caso específicamente del saber con el que se pretende dialogar, que incluye los contenidos de la educación secundaria oficial. Así, por ejemplo, las Secundarias Comunitarias integran, con motivo de fechas festivas del calendario oficial, jornadas de historia en sus actividades formativas, pero buscan cuestionar las perspectivas del relato histórico en cuestión, investigando las relaciones de poder y asimetrías sociales de trasfondo. De esta manera, uno de los educadores indica a los estudiantes "que la historia oculta es la que hay que investigar porque siempre se ha querido reprimir a los indígenas" (Ob-aula-3er grado). En una jornada de historia se busca que los jóvenes, de acuerdo con la metodología del modelo, generen preguntas a partir de las cuales abordar la Independencia de México.

El educador Carlos y los estudiantes recuperan las preguntas sobre la historia de la independencia que empezaron a elaborar ayer, como "problematización" de este episodio histórico. Generan nuevas preguntas sobre la marcha...

Isaac: Profe, quién hizo las leyes de México.

Carlos escribe la pregunta en el pizarrón.

Irina: Cuántas personas va a haber en la guerra, en la lucha.

Eneida: Cuántas personas mueren por esta guerra...

Carlos sigue escribiendo: ¿Quién implementó la América? ¿Qué significa?

Isaac: ¿Por qué los ciudadanos no quieren investigar sobre esto?

Ingrid: ¿Por qué no dicen la verdad de cómo fue en verdad la revolución?

Carlos escribe: ¿Por qué se oculta la historia verdadera?

Isaac: Eso, eso es lo que quería decir.

(escriben)

Carlos escribe: ¿Qué conflictos se han vivido desde 1810 a $1920 ?$ 
De lo comunitario a lo escolar: elementos para el debate sobre la escuela comunitaria indígena

Ingrid: ¿A quién afectaron más?

Carlos escribe: ¿En qué afectó la independencia de México?

Siguen copiando del pizarrón...

Carlos: Ustedes lo van a analizar, nosotros los vamos a estar apoyando, nada más. Van a empezar a hacer sus textos reflexivos. Implica una inteligencia del estudiante. Es la reflexión de ustedes. No les vamos a dictar, ni el libro lo van a copiar. Van a hacer sus propias reflexiones. Sí, ¿queda claro? No es un juego. Entra aquí el grito, entra en la conspiración de Querétaro (Ob-aula-3er grado).

La dimensión etnopolítica reflejada en la reflexión de los diferentes conocimientos se perfila a través del discurso de los educadores en el aula, pero también en otros espacios comunitarios. Más allá de esta mirada crítica, el diálogo con otros saberes resulta sumamente relevante para los integrantes de la comunidad, puesto que las exigencias del entorno implican también la necesidad de que los jóvenes se familiaricen con una diversidad de conocimientos.

Yo creo que sí es bueno porque dentro de esa escuela rescatan mucho lo que es la costumbre del pueblo, los usos y costumbres que traen nuestros antepasados anteriormente. Y por ejemplo ahorita tanto están aprendiendo lo nuevo que se está manejando hoy en este día, tanto están rescatando lo que se ha estado perdiendo de los abuelitos (E-Secretario-comunidad).

El planteamiento del rescate cultural tiende a localizar los saberes de la cultura originaria en el pasado; son relacionados con los conocimientos de las personas mayores, lo cual, por otra parte, indica cómo aún sigue vigente la concepción culturalmente forjada del valor de la experiencia comunitaria. Ésta es adquirida por los comuneros ${ }^{10}$ a lo largo del ejercicio de las prácticas locales productivas, organizativas, de salud, entre otras, y se constituye como una fuente de saber para el aprendizaje de las siguientes generaciones.

Por otra parte, la tendencia a asociar "lo propio" con el pasado es relacionada por algunos autores con otro proceso de esencialización. Dietz, al analizar la corriente de pensamiento indígena del comunalismo, señala cómo en algunos casos el discurso de lo comunitario se inclina a obviar la heterogeneidad presente en todo cuerpo social y reduce "'la intracultura' local a lo antiguo, lo tradicional y lo 'auténtico"' (Dietz, 1999: 356). Entiende para ello el comunalismo como modelo reivindicativo que "procura fortalecer y privilegiar los recursos endógenos... frente a la imposición de modelos exógenos y colonizadores" (Dietz, 2013: 179). Fernández y Estrada, retomando a Trollinger (2012), aluden en este mismo sentido a la nostalgia por el pasado y por la pérdida como parte de la sustancialización de la cultura propia, que aquí encontramos presente sobre todo en el

10 Entendemos por comuneros a los integrantes de la comunidad originaria; Díaz (2007) emplea también el término de comuneros-ciudadanos. La pertenencia comunitaria se relaciona tanto con derechos ciudadanos como con el cumplimiento de obligaciones (Maldonado, 2010). 
discurso de comuneros mayores, pero también en algunos casos en el de los educadores y de los estudiantes de la secundaria (Fernández y Estrada, 2014: 20).

El diálogo con lo externo, sin embargo, se ha vuelto también necesario y deseable; lo que se busca son condiciones de horizontalidad, en un difícil equilibrio frente a las influencias de la migración, la tecnología y los que han vuelto más porosos los límites del territorio comunitario hacia otro tipo de espacios, más allá de la comunidad. Conllevan transformaciones en los procesos y las prácticas culturales e identitarias, que se perciben como más marcadas en el caso de los jóvenes, abiertos a aprender y a comunicarse con el mundo, por ejemplo, a través de las redes sociales. Silva constata que "la educación formal y la escuela constituyen sólo una de las esferas en las cuales las identidades sociales son construidas... lo cultural y lo social son también dimensiones pedagógicas y curriculares" (Silva, 1997: 5); el aprendizaje, de esta manera, ocurre en diferentes espacios y dimensiones que confluyen en cómo se definen las identidades.

Estos cambios paulatinos atañen a las diferentes generaciones y requieren que la escuela comunitaria se convierta en un espacio dialógico. El concepto de meta-cultura al que alude Jiménez Naranjo (2012) aquí nos ilustra cómo las dinámicas de reflexión colectiva generadas en los proyectos de investigación pueden propiciar la construcción de conocimiento acerca de estas lógicas de transformación e hibridación de las prácticas culturales. Los educadores enfatizan la necesidad de incorporar la reflexión como una práctica formativa central que ha de impulsar paulatinamente procesos de reflexión en el ámbito comunitario. Conciben a la secundaria como "un espacio de reflexión de este conocimiento comunitario" que aporta a la comunidad, pues "tal vez, en la comunidad no se reflexiona, se vive; así, ya con la escuela, ahora se da esa reflexión" (Grupo de discusión-educadores). En la práctica cotidiana observamos cómo se promueven estos procesos.

Conrado pide a los estudiantes compartir las conclusiones a las que llegaron en cada equipo, y un equipo tiene que conjuntar desde allí.

Eneas lee de su cuaderno en español, sobre la carretera y el consumismo, que éste ya existía en cierta medida antes por la mercancía que traían en bestia; con la carretera, todo cambió, con la entrada de los comerciantes, la contaminación del medio ambiente, que ya no se usan productos naturales como antes.

Conrado: Continuamos con la mesa de Esteban.

Esteban: Grandes beneficios, grandes consecuencias. No tenían ningún tipo de contaminación, [sino los] beneficios de las plantas, fabricaban herramientas. ... Ya no se puede evitar la contaminación, el consumismo, ...también se puede reducir, pero para eso las personas tienen que entender que consumir los productos contamina nuestra comunidad.

Aristeo: Trajeron productos y vestuarios. Antes consumían quelites y guías. Poco a poco van extinguiendo este tipo de quelites que hay en la comunidad porque ya no lo conservan y ya no lo comen. ... beneficia a quienes tienen un trabajo que los obliga a salir, a quien le perjudica es a las personas que trabajan el campo. 
De lo comunitario a lo escolar: elementos para el debate sobre la escuela comunitaria indígena

Melanie: En 1984 destruyeron los árboles y por causa de eso se fue contaminando la tierra...

Conrado: Lo que no está en las planeaciones, es que van a trabajar sobre las propuestas. El ámbito 1, por mesas también van a trabajar, entonces sería propuestas que dan ustedes para ver esta parte que ustedes dicen. Ayer mencionaron que están perdiendo los valores comunitarios y eso afecta también a la naturaleza, que no se respeta. Qué es lo que ustedes proponen, qué es lo que se debe hacer (Ob-3er grado).

Los estudiantes de la secundaria, en el marco de sus proyectos, observan su contexto y analizan las prácticas comunitarias, visualizando los procesos históricos y y percatándose de los cambios ocurridos. Se perfila aquí, de manera implícita, la comprensión de la cultura como prácticas en continua transformación y en diálogo con en el entorno más amplio.

Para los habitantes de esta comunidad la cultura es cuando se realizan las fiestas tradicionales, todo esto empezó anteriormente y hasta ahora año con año la siguen venerando, hoy en día ya hubo cambios en las formas de festejar, las fiestas, por ejemplo, antes cuando festejaron las fiestas de la comunidad utilizaron tambor y flauta para la música y ahora ya festejan con músicas que utilizan instrumentos... La cultura de nuestros antepasados ya no es igual a la cultura que tenemos ahora. La cultura es todo lo que hacen las personas que viven reunidas en una sola comunidad (Trabajo escrito- 3er. grado).

La conciencia del cambio cultural se refleja en los diferentes proyectos; así, un proyecto de investigación que los educadores estructuraron junto con los jóvenes abordó la tecnología comunitaria analizando sus transformaciones históricas y presentes y teniendo en cuenta la apropiación de elementos externos (Bonfil Batalla, 2004; Rockwell, 2005), al clasificar las tecnologías observadas como "pre-hispánicas, asimiladas y modernas" (Ob-conversación informal educador).

La profundización del conocimiento comunitario por parte de los actores educativos en diálogo con otros conocimientos o prácticas culturales implica, de acuerdo al modelo, confrontación y reflexión; "la confrontación de su realidad con otras realidades y la posibilidad de encontrar coincidencias y divergencias, así como el conocimiento y aplicación de los contenidos curriculares del nivel de secundaria" (ESCI/IEEPO, 2007: 19). De acuerdo con uno de los asesores, autor zapoteco e integrante de larga experiencia en el modelo, ${ }^{11}$ la reflexión de los saberes es lo que llevará a la construcción de conocimiento.

La confrontación es cómo vamos a entender uno y otro, en qué se parece el uno al otro, hacia donde direcciona cada conocimiento, qué busca cada conocimiento hacer en el ser humano. Eso que se va a realizar... es la reflexión en esta confrontación. De esta reflexión vendrá una construcción. (E-HMS-asesor)

11 Agradezco a Hugo Miranda Segura sus aportaciones y las reflexiones compartidas que han abonado de manera relevante a mi comprensión de la complejidad de los conocimientos comunitarios y las implicaciones de un diálogo de saberes. 
El proceso de reflexión metacultural implica indagar cuál es el contexto histórico, sociocultural e incluso ideológico del que provienen los saberes. Se concibe al análisis de los conocimientos desde sus respectivos trasfondos como posibilidad de una construcción del saber nuevo. Observamos cómo los estudiantes de las Secundarias Comunitarias buscan examinar, en este sentido, las prácticas productivas de la comunidad; distinguen las afectaciones que los procesos más bien industriales que subyacen al artefacto del trapiche de fierro podría significar para el "buen vivir" (Mato, 2008) de los habitantes de la comunidad.

Queremos que las personas retomen su tecnología comunitaria como por ejemplo el trapiche de madera... ¿por qué lo están rescatando?... qué tal si en el trapiche de fierro se están mezclando con productos químicos, como podemos decir, el aceite, y el aceite cuando pasa el jugo de caña pues se mezclan. Entonces ya no va a ser un producto cien por ciento natural. Es lo que queremos rescatar (Grupo de discusión-alumnos 3er. grado). ${ }^{12}$

Desde el modelo se comprenden reflexión y aprendizaje en este diálogo de conocimientos como proceso que posibilita una mirada distinta hacia el futuro.

Pero [si] no estás reflexionando ni estás sentándote a reconstruir, y entonces no tiene futuro lo que estás haciendo... Si tú no haces esto, no estás trabajando para el futuro, estás anclado en un solo lugar pues (Obcurso-HMS-asesor).

Esta mirada de partir de lo propio para analizarlo y reflexionarlo en consideración de lo ajeno con un sentido constructivo llega a vislumbrarse en las argumentaciones de los mismos jóvenes que visualizan cómo, desde sus proyectos de investigación, es posible aportar a la reflexión del futuro de su pueblo.

Porque es un proyecto que elegimos nosotros porque tenemos el deseo de poder estudiar, y qué es lo más importante para la comunidad y que sirva para el futuro (Ob-registro-aula reunión de estudiantes).

En la práctica, el diálogo, como hemos mencionado, no está libre de la tendencia a la sustancialización, y que conlleva diferentes niveles de juicios de valor; esto sobre todo ante la necesidad de la reivindicación de lo propio, pero también frente a la comparación con lo ajeno como algo de

12 El trapiche de madera es elaborado por integrantes de la misma comunidad y con base en la materia prima local; podemos analizar que se trata de un objeto cultural, como la caña de origen externo, que "dejó de ser algo ajeno y pasó a formar parte de la cultura propia"; "ha habido una apropiación del objeto, de su significado y de la fabrilidad necesaria para construirlo; forma parte legítima de 'nuestra' cultura” (Bonfil Batalla, 2004: 124). El trapiche de fierro, en comparación, es una herramienta que algunos comuneros, en tiempos más recientes, han adquirido y traído a la comunidad para facilitar el proceso de producción; se considera como un objeto ajeno, pues no ha pasado por el mismo proceso de adaptación local y requiere de insumos y mantenimiento externos. 
lo que se ha estado generalmente excluido. En esta búsqueda de diálogo inter-cultural, por ende, se hace presente el conflicto como un reflejo de las tensiones que enfrentan las comunidades originarias en su vida cotidiana. Estas son generadas a partir de los procesos arriba descritos: la asimilación cultural promovida históricamente por instancias gubernamentales y la sociedad mayoritaria, y las asimetrías en cuanto a la pobreza y exclusión como parte de una discriminación estructural, atendida por programas asistenciales y compensatorios.

Y finalmente, están presentes las influencias del mundo cambiante y el contacto con las tendencias del desarrollo global a través de los medios y una serie de interrelaciones con otros espacios sociales, por la migración, la introducción de productos y hábitos de consumo provenientes de la sociedad industrializada, entre otros aspectos. Un punto relevante es cómo estas tendencias inciden en las expectativas comunitarias hacia la institución escolar y el futuro de los jóvenes, implicando las aspiraciones -justificadas y justas, y no por ello excluyentes de la revaloración de lo propio- a la inclusión en el mundo nacional y global, las posibilidades del empleo remunerado, y la validación de los logros escolares, sociales y económicos en condiciones de equidad.

Esta situación compleja ha conllevado justamente una necesidad de la reflexión comunitaria acerca de los aprendizajes deseables para los estudiantes de la secundaria -frente al modelo socialmente legitimado de la escolarización clásica-, acerca de la viabilidad y utilidad de "aprender lo propio", y también la revaloración, por parte de las generaciones jóvenes, de las prácticas comunitarias, del trabajo campesino y sus conocimientos. Esta hibridez contextual, aun a distancia de la capital de estado y del ámbito citadino, se delinea como una constelación sumamente significativa que requiere de una continua actualización y atención permanente por parte de los actores de un modelo educativo innovador como las Secundarias Comunitarias, teniendo en cuenta la misma concepción metodológica y pedagógica de la propuesta que enfatiza el carácter dialógico y participativo del aprendizaje. También es necesario visualizar, ante estas configuraciones socioculturales, cómo la vinculación entre escuela y comunidad y el diálogo entre prácticas culturales atañe a dimensiones múltiples; en este caso, queremos retomar el aspecto de las prácticas pedagógicas.

\section{Aproximaciones al diálogo de prácticas pedagógicas escolares y comunitarias}

El diálogo entre la escuela y la comunidad no se reduce a lo que comúnmente se considera como contenidos, sino que abarca también una variedad de interrelaciones y prácticas que constituyen una manera importante de vinculación y de resignificación de la interculturalidad en educación. Entre estas prácticas de interrelación entre cultura escolar y cultura comunitaria algunas ocurren sin figurar entre las estrategias explícitas; con Jiménez Naranjo (2009) las llamaríamos clandestinas. En estos intersticios de la cotidianidad, también se construyen aprendizajes, aunque de maneras tal vez poco usuales o discontinuas (Roy, 2003: 13). Otras acciones de vinculación son intencionales, pues el modelo pretende fomentar una nueva articulación entre el espacio escolar y el comunitario, superando su división histórica. 
A continuación, analizaremos cómo puede resignificarse la concepción de la cultura como prácticas sociales en relación con las mismas formas pedagógicas que se observaron en las actividades educativas de la Secundaria Comunitaria. Esta articulación no está presente siempre en la cotidianidad escolar, sino que se entreteje en las configuraciones la práctica de algunos de los educadores. Se relaciona, por una parte, con los objetivos pedagógicos del modelo; por otra, se nutre de la misma biografía de los docentes, siendo ellos mismos, aunque de otras localidades, de origen comunitario.

En la vida comunitaria y familiar, la participación de niños y niñas en las actividades familiares como parte de los procesos de aprendizaje implica el desarrollo de una autonomía temprana, con responsabilidades propias y sucesivas en las tareas que forman parte del proceso de socialización y paulatina integración a las dinámicas comunitarias y familiares. Bertely, Saravi y Abrantes constatan que los adolescentes indígenas generalmente son más autónomos, sin por ello desvincularse del entorno social; participan responsablemente, "asumiendo nuevos derechos y obligaciones vinculados a entidades colectivas como la familia o la comunidad" (Bertely, Saravi y Abrantes, 2013: 16).

En el contexto de la comunidad zapoteca en el que se realizó la presente investigación, observamos cómo los estudiantes de la secundaria se involucran en las tareas cotidianas de sus familias. No se desligan de las actividades sociales y productivas que forman parte de los procesos de socialización comunitaria. Para permitir la continuidad de esta participación y trascender las rupturas introducidas por la educación escolarizada en el aprendizaje comunitario, los educadores de la Secundaria buscan flexibilizar, en cierta medida, los tiempos escolares, además de entrelazar los conocimientos, propios y ajenos. Esta apertura conlleva el diálogo entre los sitios del aprendizaje escolar y comunitario, y propicia la significación de los temas abordados en la secundaria.

De cuando sacó panela Ingrid, y esta Lara, Isaac, llegaron a pedir permiso de estar un día. No, digo, a ver, cuántos días vas a sacar panela. Ah, toda la semana. Ah, vete toda la semana. Y ahí hicieron las redacciones y trabajaron en sus ranchos y finalmente hicieron un reporte familiar... Y ya nosotros íbamos visitando estos ranchos, fuimos en la tarde, y estaban trabajando (Grupo discusión-educador).

Bertely analiza la relación entre los procesos de socialización de una comunidad mazahua y las dinámicas de enseñanza y aprendizaje en una escuela primaria. Retomamos de su trabajo, para este artículo, sólo un aspecto específico, relacionado a la vinculación escuela-comunidad: la nodireccionalidad del trabajo áulico. La autora destaca la realización de "actividades paralelas... sin contar con el control exhaustivo por parte del maestro", empleándose de esta manera estrategias de enseñanza "no centralizadas" (Bertely, 1992: 107-108). Con ello el docente, o bien retoma algunas formas propias de la socialización comunitaria, o bien éstas ocurren sin intencionalidad.

Las Secundarias Comunitarias promueven como uno de sus rasgos el trabajo autónomo del estudiante. Es una dinámica nueva para los jóvenes, pues en los niveles escolares previos -de 
educación preescolar y primaria- la enseñanza se estructura de manera más vertical. En la práctica educativa de la secundaria se observa cómo se fomenta procesualmente que los estudiantes asuman la responsabilidad de su propio aprendizaje. En el trabajo áulico, la presencia continua del maestro no es indispensable; una vez clarificadas las tareas, los jóvenes realizan colaborativamente diferentes actividades, de acuerdo a sus avances. El docente proporciona orientación cuando ésta es requerida.

Los chicos están trabajando geografía. Están al parecer en las binas por mesas y el educador Conrado les pide que mencionen las coordenadas de los dos países que escogieron. En el pizarrón hay dos mapas colgados, uno de América, otro del resto del mundo... Alexander le contesta a Conrado en zapoteco cuando éste le dice algo en español. Las tres chicas (con excepción de Nadia) están leyéndose mutuamente al parecer un cuento en español, se turnan el texto y leen en voz alta. Conrado sale. Le toca a Guadalupe leer. Conrado regresa con una libreta y se sienta. No interfiere con la dinámica de los estudiantes. Claudio y Lalo hablan en zapoteco sobre los océanos, pacífico y atlántico. Lalo algo dice a Alexander en zapoteco. Ismael está revisando una hoja blanca con un mapa dibujado. Nadia le lleva su mapa a Conrado y le pregunta algo. Conrado en el mapita le explica algo sobre las coordenadas (Ob-aula-1er grado).

Los educadores impulsan a los estudiantes a resolver, ellos mismos, las dudas que emergen en la investigación, como parte de la formación para el aprendizaje autónomo; proporcionan pocas explicaciones o respuestas directas, pues asumen que el estudiante ha de construir activamente sus aprendizajes y que el trabajo por proyectos y la investigación se aprenden a través de la práctica.

Estudiante: Casi cuando explica, explica como una sola vez. Y en la primaria sí repite y repite si entendimos o no. Nos preguntan, niños, ¿entendieron? Nosotros contestamos que sí o no, y si decimos no, vuelven a explicar otra vez y así hasta entender porque dicen que es necesario... Los profes tienen que explicar para que así nosotros también vayamos aprendiendo más...

Investigadora: Y aquí ya no explica tanto el maestro.

Estudiante: Tiene que ser uno, tiene que investigar y tiene que hacer su redacción, así (E-Bárbara-1er grado).

En el contexto comunitario y familiar nos damos cuenta de que los procesos de aprendizaje en la primera infancia ocurren por medio de dinámicas similares. No se impulsa de manera directa la participación activa en las labores familiares, sino que aprendizaje y participación se dan por iniciativa de los niños, a partir de la observación y la imitación del ejemplo proporcionado por los otros integrantes de la familia, inmersos en el trabajo y sus locaciones cotidianas.

Cuando entré y comenté, "están desgranando maíz", [la joven] dijo, "aprovechando el tiempo", y doña Estrella [la abuela] comentó que además estaba dando el ejemplo a sus hijas. Y ya que empecé a observar 
la escena, me llamó la atención que la pequeña de cuatro años estaba distraídamente desgranando mazorcas con sus minúsculas manos en una palangana de plástico y tomando más mazorcas del costal blanco de rato en rato... Pregunté a la joven mamá si ella le había enseñado o dicho a su hija, y me contestó que no, que así nomás ella lo está haciendo, que no le ha dicho nada. Y que lo mismo hace con el café, sin que se le haya dicho nada, se pone a hacerlo. Comenté que lo observaba y por eso lo hacía, y la mamá asintió (Ob-casa de doña Estrella).

Aunque en la práctica de las Secundarias se presenten tensiones relacionadas con la experiencia escolar previa de los jóvenes, vislumbramos cómo algunos docentes, originarios de otras comunidades zapotecas y con larga trayectoria en el modelo, logran dar cierta continuidad a las formas de aprendizaje comunitarias. Generan dinámicas de interacción áulicas y extra-áulicas poco directivas, para fomentar procesos de aprendizaje más autónomos. Son pacientes y buscan no forzar la participación, pues comprenden la formación de los jóvenes en estos nuevos hábitos como un proceso.

El educador Conrado organizó... ensayos arriba en la cancha, todo el programa de teatro y bailables. Los muchachos anduvieron muy dispersos. Conrado estuvo muy paciente, no forzó nada. Les decía, segunda llamada, tercera llamada, pero no todos se aparecieron ni participaron... Conrado a una de sus alumnas le dijo que él estaba a disposición, ya dependía de ellos mismos. A mí me contó que cuando sus alumnos [de tercero] empezaron, también había algunos muy inquietos, y que fue todo un proceso. Que él les dio tiempo, poco a poco. Y ahora en tercero el resultado del proceso fue que para él ha sido mucho menos trabajo, ya trabajan de manera muy independiente (Ob-cancha).

Las dinámicas de los procesos de aprendizaje poco directivos en la práctica de las Secundarias Comunitarias se desarrollan entre tensiones; se presentan algunas contradicciones en el quehacer educativo cotidiano, además de cuestionamientos comunitarios ante esta forma pedagógica. Diferentes factores intervienen, entre ellos un aspecto relevante consiste en el imaginario de "la educación escolar" que ha sido socialmente interiorizado y se refleja en los discursos de diferentes actores, pero también en las trayectorias de los mismos docentes formados en instituciones de corte tradicional.

Otro factor radica en el valor comunitario del respeto como parte de la comunalidad oaxaqueña (Martínez Luna, 2010), y que tradicionalmente media las interrelaciones sociales en las comunidades, y específicamente el trato a los mayores como poseedores de experiencia. El respeto se ha visto afectado por las influencias del contacto con otros espacios culturales, como la migración urbana y los medios de comunicación, además de la misma escolarización. Interpretamos en relación con ello las percepciones que tienen algunos padres de familia de las dinámicas formativas de la Secundaria: 
De lo comunitario a lo escolar: elementos para el debate sobre la escuela comunitaria indígena

Como no está el maestro, luego bien contentos están peleándose, jugando, ya no se están aprendiendo, ya no se están en su salón, salen... Y luego ahorita que ya no hay mucha disciplina también (E-Eréndira-madre de familia).

En la práctica, los estudiantes y educadores transitan entre espacios de aprendizaje a través del diálogo con el conocimiento comunitario, con las entrevistas comunitarias realizadas por los estudiantes a "portadores de conocimiento comunitario", recuperando las vías tradicionales de transmisión oral del saber entre generaciones (Briseño, 2015) y las visitas a observar en el lugar de las prácticas. El hecho de trascender en las actividades formativas los límites del perímetro escolar contrasta con la gramática escolar clásica (Tyack y Cuban, 1995); aunque nosotros interpretemos esta flexibilidad en términos del diálogo entre prácticas y del aprendizaje situado (Lave y Wenger, 1991; Sagástegui, 2004), es percibida como problemática por algunos comuneros.

Andan investigando, y andan en casa por casa, a veces ahí andan, hacen maldad... Mucho problema buscan porque es que tienen que estar en el salón, tienen que aprender algo, las cuentas, español. Y puro zapoteco, hasta con los maestros, no, casi no hablan español (E-Eréndira madre de familia).

La idea de la escuela tradicional que ha sido interiorizada por muchos, se asocia con el acceso a los saberes legitimados por la sociedad mayoritaria y el aprendizaje en el aula y a través de los libros, bajo la dirección del docente. La propia experiencia escolar de muchas madres y padres de familia en la escuela primaria se rigió por las lógicas más verticales de las políticas de asimilación e integración cultural; en los relatos destacan la disciplina rígida y la discriminación a través de la exclusión de la lengua originaria del espacio formativo.

Yo cuando estudié en la primaria, ahí sí no podías faltar, no podías llegar tarde porque los maestros te castigaban, te pegaban con varitas. Y ya cuando mis hijas entraron creo que ya no, ya habían quitado eso... cuando yo estudié, los maestros no querían que habláramos zapoteco, sino que español porque si hablábamos zapoteco pagábamos 50 centavos (E-Griselda-madre de familia).

Ha de resaltarse que las Secundarias Comunitarias pretenden aportar a un proceso de deconstrucción, tanto de las experiencias históricas de exclusión y discriminación, como de las significaciones atribuidas a la formación escolar a partir de un currículum nacional de implementación centralizada. Proponen, frente a ello, formas pedagógicas más flexibles, comunicadas y co-ejercidas, que permiten resignificar localmente el sentido de la institución escolar y fortalecer la cultura comunitaria a partir del diálogo necesario entre "lo propio" y "lo ajeno". 


\section{Conclusiones}

La experiencia descrita representa un esfuerzo importante por concebir y poner en práctica, ante un panorama de relaciones interculturales asimétricas, históricamente conformadas, y en una sociedad marcada por sus condiciones desiguales y excluyentes, una propuesta educativa que pone en el centro el diálogo entre escuela y comunidad, pero también busca, desde la práctica, generar insumos para la necesaria interlocución con otras culturas y especialmente con la sociedad mayoritaria. Se inserta, de esta manera, en el debate de la interculturalidad en educación a partir del replanteamiento de la gramática clásica de la educación escolarizada (Tyack y Cuban, 1995). Propone un currículum flexible, articulado con las necesidades, las voces y las prácticas comunitarias; así, los contenidos del aprendizaje llegan a reflejar los procesos socioculturales, las problemáticas variadas, y las tensiones entre "lo propio" y "lo ajeno" que viven la comunidad y sus habitantes.

Al plantearse desde la reivindicación de las epistemologías propias y buscar el diálogo horizontal con los conocimientos disciplinarios, el modelo se sitúa en el difícil ejercicio de la articulación de saberes y prácticas procedentes de distintos contextos socioculturales, con diferentes racionalidades. A través de los proyectos de investigación que estructuran procesualmente las actividades formativas se generan procesos de aprendizaje que se vinculan con la experiencia de los estudiantes, con la biografía comunitaria y con sus prácticas cotidianas. Requiere, además, que los jóvenes generen preguntas acerca de su contexto y aprendan a observarlo. Esta cercanía con el mundo de vida de los actores aporta a la significación del aprendizaje, de los jóvenes y de la comunidad.

Las interrelaciones entre el modelo y las expectativas comunitarias, sin embargo, son marcadas por ciertas tensiones; por una parte, se vislumbra el anhelo de la reivindicación de la cultura e identidad propias; por otra, padres y jóvenes demandan una formación que les permita enfrentar los retos del contacto con otras realidades sociales y posibilite una participación horizontal -académica, laboral y social- en la sociedad mayoritaria. Los procesos de hibridación, apropiación cultural y resistencias a lo largo de la historia de las comunidades configuran un escenario del aprendizaje que no admite planteamientos educativos unilineales. Requiere de la sensibilidad para dialogar con las preocupaciones, los intereses, pero también los imaginarios de los actores, con las diferencias generacionales cada vez más presentes a través de la incidencia de los medios, el internet, la televisión, entre otros, que son fuente de aprendizajes para los niños y jóvenes. La atención a las inquietudes emergentes y la pluralización de las identidades, sin menoscabo del cuidado y la continuidad de las prácticas culturales propias, son una exigencia compleja para la conformación de una escuela distinta.

Se requiere formar a jóvenes que actúen de manera constructiva en su propia realidad social; esta tarea conlleva diferentes dimensiones. Además de la profundización y reflexión de los conocimientos comunitarios, reposicionando la relevancia y vigencia de las prácticas locales, también han de abordarse las problemáticas y necesidades que caracterizan el contexto como espacio en 
De lo comunitario a lo escolar: elementos para el debate sobre la escuela comunitaria indígena

comunicación. Las Secundarias Comunitarias nos muestran cómo la escuela debe abrirse hacia el mundo circundante, local y más amplio; para ello se requiere de una mirada compleja del contexto y de los procesos culturales, reto que poco ha cumplido la escuela tradicional al buscar la interculturalización de su currículum (Jiménez Naranjo, 2009, 2012).

Las Secundarias Comunitarias nos proporcionan pautas para la reflexión acerca de nuevas continuidades y necesarias articulaciones de escuela y comunidad: entre aprendizajes comunitarios y escolares, pero también entre pedagogías familiares, culturalmente forjadas, y estrategias docentes. Esta vinculación de prácticas permite que los jóvenes no se desliguen de su entorno y que se suavicen las rupturas que la escolarización de la educación ha introducido en los procesos de socialización de las comunidades originarias.

Los planteamientos educativos de esta propuesta alternativa no se alejan, aparentemente, de los discursos actuales de la formación para el auto-aprendizaje, la resolución de problemas, la interdisciplinariedad; de una educación para la vida, la ciudadanía participativa y para la diversidad. Lo que no hemos de perder de vista es el trasfondo de la exclusión social y los procesos históricos de marginación que hacen que subyazca un importante posicionamiento político al quehacer educativo de este $-\mathrm{y}$ otros- modelos diferenciales.

La dimensión de la reflexión metacultural de los procesos culturales y sociales fomentada en las Secundarias contiene, de esta manera, el potencial de un análisis crítico de los fenómenos sociales observados en la investigación, de los discursos y los contenidos escolares, y, no menos importante, de la propia práctica y discursividad como propuesta educativa que se perfila como ejemplo para muchos actores de la educación indígena en Oaxaca.

Señalamos como una de las aportaciones del modelo la apuesta por la permeabilidad de la institución escolar a las dinámicas inter-culturales de su entorno y en las que se encuentran inmersos los estudiantes, padres de familia y la misma comunidad, reconociendo también las dimensiones plurales, conflictivas y contradictorias de la realidad social. Ante estas constelaciones, resalta el valor de una pedagogía más flexible, dialógica y co-ejercida. Las Secundarias Comunitarias invitan a repensar la institución escolar como espacio constructivo, en interacción con su contexto y sus actores, para generar propuestas que promuevan aprendizajes pertinentes y necesarios para los jóvenes como integrantes y futuros ciudadanos de sus comunidades y de la sociedad más amplia.

\section{Bibliografía}

Barba, A., R. Landey, y O. Hernández (2014). "La alternativa educativa intercultural wixárika". En: Luengo, E. (coord.). Las alternativas ciudadanas para otros mundos posibles: pensamiento y experiencias. Guadalajara: ITESO. pp. 53-70. Recuperado de: http://formacionsocial.iteso. $\mathrm{mx} /$ documents/10901/0/Complexus+5+noviembre/2e1e4f7b-4788-4d9c-a6cb-7f531c5943c5 
Baronnet, B. (2009). "Autonomía y educación indígena: Las escuelas zapatistas de las cañadas de la Selva Lacandona de Chiapas, México". Tesis doctoral. El Colegio de México y Universidad La Sorbonne.

Bensasson, L. (2013). “Educación intercultural en México ¿por qué y para quién?”. En: Baronnet, B. y M. Tapia (coords.). Educación e Interculturalidad. Política y políticas. México: UNAM/ CRIM. Recuperado de: http://www.libros.unam.mx/digital/V7/51.pdf

Bertely, M. (1992). "Adaptaciones docentes en una comunidad mazahua". Nueva Antropología, (42). México.

_ (2005). "¿Apropiación escolar o etnogénesis? La escuela federal y socialista en una villa zapoteca mexicana (1928-1940)". En: Sociedad Mexicana de Historia de la Educación. Memoria, conocimiento y utopía. México: Pomares.

—_ (2011). "Aprendizajes, (inter) aprendizajes, (re) aprendizajes y (des) aprendizajes al tejer una red de cuatro puntas. De cómo se vive la interculturalidad al pescar conocimientos y significados indígenas y comunitarios". En: Bertely, M. (ed.). Interaprendizaje entre indigenas. De cómo las y los educadores pescan conocimientos y significados comunitarios en contextos interculturales. México: CIESAS-UPN, pp. 9-38.

— G. Saraví y P. Abrantes (2013). Adolescentes indígenas en México: derechos e identidades emergentes. México: CIESAS/UNICEF.

Briseño, J. (2015). "Entrevistas comunitarias, la recuperación del conocimiento comunitario desde la escuela. El caso de las Secundarias Comunitarias Indígenas, Oaxaca, México". En: Archivos Analíticos de Políticas Educativas, 23 (95). Recuperado de: http://dx.doi.org/10.14507/epaa. v23.2081

Bonfil Batalla, G. (1991). "La teoría del control cultural en el estudio de procesos étnicos". En: Revista Estudios sobre las Culturas Contemporáneas IV (12) 165-204. México: Universidad de Colima. Recuperado de: http://www.redalyc.org/pdf/316/31641209.pdf

— (2004). "Pensar nuestra cultura". En: Revista Diálogos en la acción, primera etapa. México: Dirección General de Culturas Populares e Indígenas. Recuperado de: http://www.ilam.org/ ILAMDOC/sobi/Guillermo\%20Bonfil_Pensarnuestracultura-Pat.pdf

Castro-Gómez, S. (2000). "Ciencias Sociales, violencia epistémica y el problema de la 'invención del otro"”. En: Lander, E. (ed.). La colonialidad del saber: eurocentrismo y ciencias sociales. Perspectivas latinoamericanas. Buenos Aires: CLACSO, pp.145-161.

CNEM (2013). Educación propia: Pueblos Maya, Xinca y Garifuna. Lineamientos de construcción de políticas y de diseño y aplicación curricular. Guatemala: Ajpop Mayab’Tijonik CNEM. Recuperado de: http://www.infoindigena.org/images/Publicaciones_generales/Educacion/Port_Educacion_ Propia.pdf 
De lo comunitario a lo escolar: elementos para el debate sobre la escuela comunitaria indígena

De Aguinaga, R. (2010). Tatuutsi Maxakwaxí. Una experiencia indigena de educación autonómica. Guadalajara: ITESO.

Díaz, F. (2007). Escrito. Comunalidad, energía viva del pensamiento mixe. México: UNAM.

Díaz Barriga, F. (2003). "Cognición situada y estrategias para el aprendizaje significativo". En: Revista Electrónica de Investigación Educativa, 5 (2). Recuperado de: http://redie.ens.uabc. $\mathrm{mx} / \mathrm{vol} 15 \mathrm{no} 2 /$ contenido-arceo.html

Díaz de Rada, A. (2011). El taller del etnógrafo. Materiales y herramientas de investigación en etnografía. Madrid: UNED.

Dietz, G. (1999). La comunidad purhépecha es nuestra fuerza. Etnicidad, cultura y región en un movimiento indígena de México. Quito: Abya Yala.

— y L. Mateos (2008). "El discurso intercultural ante el paradigma de la diversidad: estructuraciones subyacentes y migraciones discursivas del multiculturalismo contemporáneo". En: Bastos, S. Multiculturalismo y futuro en Guatemala. Guatemala: FLACSO/OXFAM.

— (2011). Interculturalidad y educación intercultural en México: un análisis de los discursos nacionales e internacionales en su impacto en los modelos educativos mexicanos. México: CGEIB.

(2013). "Comunidad e Interculturalidad entre lo propio y lo ajeno: hacia una gramática de la diversidad”. En: Baronnet, B. y M. Tapia (coords.). Educación e interculturalidad. Política y políticas. México: UNAM/CRIM. Recuperado de: http://www.libros.unam.mx/digital/V7/51.pdf

Escárcega Zamarrón, S. (2009). "Trabajar haciendo: activist research and interculturalism". En: Intercultural Education, 20(1), pp. 39- 50.

ESCI/IEEPO (2007). Secundaria para la atención de comunidades pertenecientes a los pueblos originarios en el estado de Oaxaca (versión sintética). Oaxaca: ESCI/IEEPO.

Fernández Repetto, F. e I. Estrada Burgos (2014). "Esencialización y espectacularización de lo maya. Turismo voluntario y étnico en una comunidad yucateca". En: Península [online]. 9(1) 9-32. Recuperado de: http://www.scielo.org.mx/pdf/peni/v9n1/v9n1a1.pdf

Fundación Promigas (2010). Educación propia: una experiencia de construcción colectiva con comunidades wayuu. Colombia: Editorial Fundación Promigas/Fundación Caminos de Identidad. Recuperado de:

http://www.fundacionpromigas.com.co/wps/wcm/connect/43695f00445f396685cdff82af99c 9ea/EDUCACIONPROPIAWEB+\%282\%29.pdf?MOD=AJPERES

García Canclini, N. (2001). Culturas híbridas. Estrategias para entrar y salir de la modernidad. Barcelona: Paidós.

Gasché, J. (2004). "Hacia una propuesta curricular intercultural en un mundo global". Ponencia. En: Rodríguez, M. A. (comp.). Foro Latinoamericano de Interculturalidad, Educación y Ciu- 
dadanía. Cuetzalán, México: SEP-CGEIB, Contracorriente, Observatorio Ciudadano de la Educación, FLAPE, pp. 177-200.

Giménez, Gilberto (2000). "Identidades étnicas: estado de la cuestión”. En: Reina, Leticia. Los retos de la etnicidad en los Estados-nación del siglo XXI. México: CIESAS, INI, Miguel Ángel Porrúa.

(2009). "Cultura, identidad y memoria: Materiales para una sociología de los procesos culturales en las franjas fronterizas". En: Frontera norte, 21(41), 7-32. Recuperado de: http:// www.scielo.org.mx/scielo.php?script $=$ sci_arttext\&pid $=$ S0187-73722009000100001\&lng=es \&tlng=es

González Apodaca, E. (2006). "Etnicidad, intermediación y escuela: tres iniciativas de bachillerato intercultural en la región mixe". Tesis doctoral. México: UAM-Ixtapalapa.

— y A. Rojas (2013) "Proyectos locales, autonomía educativa y resistencia indígena". En: Bertely, M., G. Dietz y G. Díaz Tepepa (coords.). Multiculturalismo y Educación 2002-2011. Col. Estados de conocimiento. México: ANUIES, COMIE, pp. 383-413.

Hamel, R. (2001). "Políticas del lenguaje y educación indígena en México. Orientaciones culturales y estrategias pedagógicas en una época de globalización". En: Bein, R. y J. Born (eds.). Políticas lingüisticas. Norma e identidad. Buenos Aires: UBA, pp. 143-170.

Jiménez Naranjo, Y. (2009). Cultura comunitaria y escuela intercultural. México: CGEIB, SEP. (2012). "Cultura escolar y cultura comunitaria: una perspectiva intercultural en la construcción curricular". Ponencia presentada en el IAIE. Veracruz.

Lave, J. y E. Wenger (1991). Situated Learning. Legitimate Peripheral Participation. Nueva York: Cambridge University Press.

Leff, E. (2004). Racionalidad ambiental. La reapropiación social de la naturaleza. México: Siglo XXI.

López Pérez, A. (2004). "Individuo y comunidad: imágenes identitarias y tejidos culturales en los proyectos educativos de México". En: Gómez Sollano, M. y B. Orozco (2004). Espacios Imaginarios y sujetos de la Educación en la. Transición Epocal. México: Plaza y Valdés, pp.189-218.

Maldonado, B. (2010). "Comunidad, comunalidad y colonialismo en Oaxaca, México. La nueva educación comunitaria y su contexto". Tesis doctoral. Países Bajos: Universidad Leiden.

Martínez Luna, J. (2010). “Cotidianidad y comunalidad”. En: Homo Erectus. Revista de educación y cultura política, año 1, (1) 21-25.

_ (2013). "Textos sobre el camino andado". Tomo 1. Oaxaca: CAMPO-CSEIIO-CMPIO-PLAN PILOTO-CNEII-CEEESCI.

Martínez Buenabad, E. (2015). "La educación intercultural y bilingüe (EIB) en México. ¿El camino hacia la construcción de una ciudadanía democrática?” Revista Relaciones (141), pp. 103-131. 
De lo comunitario a lo escolar: elementos para el debate sobre la escuela comunitaria indígena

Recuperado de:

http://www.colmich.edu.mx/relaciones25/files/revistas/141/pdf/05_Elizabeth_Martinez.pdf

Mato, D. (2008). "No hay saber 'universal', la colaboración intercultural es imprescindible". En: Alteridades, 18 (35) 101-116. México: UAM-Iztapalapa.

Pieck, E., G. Messina y Colectivo docente (2008). Nuestras historias. El lugar del trabajo en las Telesecundarias Vinculadas con la Comunidad. México: Universidad Iberoamericana.

PRATEC (2004). Una escuela amable con el saber local. Perú: PRATEC. Recuperado de: http://www. pratecnet.org/pdfs/Una\%20escuela\%20amable.pdf

Rendón Monzón, J. J. (ed.) (2003). La comunalidad. Modo de vida en los pueblos indios. México: CONACULTA, pp. 91-108.

_ (2011). La flor comunal. Explicaciones para interpretar su contenido y comprender la importancia de la vida comunal de los pueblos indios. Oaxaca: CNEII, CMPIO, CEEESCI, CSEIIO.

Rengifo, G. (2003). La enseñanza es estar contento. Educación y afirmación cultural andina. Lima. Perú: Proyecto Andino de Tecnologías Campesinas (PRATEC).

Richard, N. (1993). "Alteridad y descentramiento culturales". En: Revista Chilena de Literatura, (42) 209-215. Chile: Universidad de Chile. Recuperado de: http://19bienal.fundacionpaiz.org. gt/wp-content/uploads/2014/02/Nelly-Richard-Alteridad-y-descentramiento-culturales.pdf

Rockwell, E. (2005). "La apropiación, un proceso entre muchos que ocurren en ámbitos escolares". En: Memoria, conocimiento y utopía. Anuario de la Sociedad Mexicana de Historia de la Educación. (1), pp. 28-38. Barcelona: Pomares.

— (2011). La experiencia etnográfica. Buenos Aires: Paidós.

Rogoff, B., L. Moore, M. Correa-Chávez y A. Dexter (2015). "Children develop cultural repertoires through engaging in everyday routines and practices". En: Grusec, J. y P. Hastings (eds.). Handbook of Socialization. Theory and Research. Nueva York: Guilford, pp. 472-498. Roy, K. (2003). Teachers in Nomadic Spaces: Deleuze and Curriculum. Nueva York: Peter Lang. Sagástegui, D. (2004). "Una apuesta por la cultura: el aprendizaje situado”. En: Sinéctica (24), pp. 30-39.

Santos, B. (2007). "Beyond Abyssal Thinking: From Global Lines to Ecologies of Knowledges". En: Review. Recuperado de: http://www.boaventuradesousasantos.pt/media/Beyond_Abyssal_Thinking_Review_2007. pdf

— (2009). Descolonizar el saber, reinventar el poder. Montevideo: Trilce.

— (2010). Para descolonizar Occidente: más allá del pensamiento abismal. Buenos Aires: CLACSO/Prometeo Libros.

Sartorello, S. (2009). "Una perspectiva crítica sobre interculturalidad y educación intercultural bilingüe: El caso de la Unión de Maestros de la Nueva Educación para México (UNEM) y edu- 
cadores independientes en Chiapas". En: Revista Latinoamericana de Educación Inclusiva, 3(2), pp. 77-90. Recuperado de: http://www.rinace.net/rlei/numeros/vol3-num2/art5.pdf

Silva, T. (1997). "Descolonizar el currículo. Estrategias para una pedagogía crítica". En: Revista Crítica Educativa. Año II (2), Buenos Aires.

Tovar, M. (2009). "Interculturalidad y epistemología". Ponencia en el II Congreso Internacional y VII Seminario Nacional de Investigación en Educación, Pedagogía y Formación Docente. Medellín: Universidad de Antioquia/Universidad Pedagógica Nacional de Colombia. Recuperado de:

http://cursos.campusvirtualsp.org/pluginfile.php/3251/mod_page/content/1/determinantes/bibliocomplementaria/unidad_3.1bc/ud31bc_interculturalidad_y_epistemologia_MTovar.pdf

Tyack, D. y L. Cuban (1995). Tinkering Towards Utopia: A Century of Public School Reform. Cambridge: Harvard University Press

Velasco, H. y A. Díaz de Rada (1997). La lógica de la investigación etnográfica. Un modelo de trabajo para etnógrafos de escuela. Madrid: Trotta.

Velasco, S. y A. Jablonska (coord.) (2010). Construcción de políticas educativas interculturales en México: debates, tendencias, problemas, desafios. México: UPN.

Viaña, J. (2008). "Reconceptualizando la interculturalidad". En: Mora, D. y S. De Alarcón. Investigar y Transformar. Reflexiones sociocríticas para pensar la educación. La Paz: Instituto Internacional de Integración, pp. 293-344.

Walsh, C. (2005). La interculturalidad en la educación. Perú: Ministerio de Educación/UNICEF.

Recibido: 14/04/16

Dictaminado: 09/06/16

Corregido: 28/06/16

Aceptado: 11/07/16 